АБДУРАЗАКОВ Магомед Мусаевич

Центр теории и методики обучения математике и информатике

Институт стратегии развития образования Российской академии образования

г. Москва, Россия

abdurazakov@inbox.ru

НИМАТУЛАЕВ Магомедхан Магомедович Финансовый университет при правительстве РФ г. Москва, Россия

АСКЕРОВ Алаутдин Садитдинович

Дагестанский государственный педагогический университет

г. Махачкала, Россия

ОБРАЗОВАТЕЛЬНАЯ СРЕДА КАК

ДИДАКТИЧЕСКОЕ УСЛОВИЕ ОБЕСПЕЧЕНИЯ ЭФФЕКТИВНОСТИ ОБУЧЕНИЯ И РАЗВИТИЯ личности

Информатизация образования рассматривается авторами как единый структурный процесс достижения целей образования через посредство глобальной информатизации общества, выражения и развития ее достижений в образовании. К теории и методике обучения информатике в школе и методической системе обучения информатике в вузе информатизация образования имеет самое непосредственное отношение. Обучение информатике предполагает его опору на ресурсы информационно-образовательной среды, наличие достаточного количества электронных образовательных ресурсов информатизации образования. При этом методическая система обучения, реализуемая и развиваемая в новой информационнообразовательной среде, приобретает новые черты, новый характер взаимодействия своих компонентов. Как известно, любой образовательный процесс всегда проходит в определенной образовательной среде, формируемой субъектами этой среды (преподаватель, обучаемые), организационными формами обучения, средствами обучения и многими другими компонентами. В работе роль и место информационно-образовательной среды определяется с позиции эффективности, результативности и качества образования и, следовательно, эффективности и качества их взаимного воздействия на принципах прямой и обратной связи. С этих же позиций оценивается ее состояние и развитие. Соответственно этому рассматривается выражение этой взаимосвязи в методической системе обучения информатике, в ее базовых компонентах.

Ключевые слова: информатика, обучение, методическая система обучения, информатизация, содержание, средства, ресурсы, информационнообразовательная среда.
Magomed M. ABDURAZAKOV

Center of Theory and Methodology of Teaching

Mathematics and Informatics

Institute for Strategy of Education Development of the Russian Academy of Education Moscow, Russia abdurazakov@inbox.ru

Magomedhan M. NIMATULAEV Financial University under the Government of the Russian Federation Moscow, Russia

Alautdin S. Askerov, Dagestan State Pedagogical University Makhachkala, Russia

\section{EDUCATIONAL ENVIRONMENT AS A DIDACTIC CONDITION FOR ENSURING THE EFFECTIVENESS OF EDUCATION AND PERSONAL DEVELOPMENT}

The authors consider the informatization of education as a single structural process of achieving the goals of education through the medium of global informatization of society, the expression and development of its achievements in education. To the theory and methodology of teaching computer science in the school and the methodological system of teaching computer science in the university, the informatization of education is most directly related. Training in informatics implies its reliance on the resources of the information and educational environment, the availability of a sufficient number of electronic educational resources for the informatization of education. At the same time the methodical system of education, implemented and developed in the new information and educational environment, acquires new features, new essence of its components interaction. It is well known, that any educational process always takes place in a certain educational environment, formed by the subjects of this environment (teacher, trainees), organizational forms of education, teaching aids and many other components. In the work, the role and place of the information and educational environment is determined from the standpoint of the effectiveness, results and quality of education and, consequently, the effectiveness and quality of their mutual impact on the principles of forward and backward linkages. From the same positions, its state and development are assessed. Accordingly, we consider the expression of this relationship in the methodical system of teaching computer science, in its basic components.

Keywords: informatics, training, methodical system of education, informatization, content, means, resources, information and educational environment. 
Сегодня достижение современного качества образования является главной задачей государственной образовательной политики. Это обусловлено развитием образования, его требований и потребностей; стремлением к поиску средств, форм и методов их реализации. Как отмечается в «Концепции Федеральной целевой программы развития образования на 2016-2020 годы» «...должны быть решены задачи достижения высокого стандарта качества содержания и технологий для всех видов образования - профессионального (включая высшее), общего и дополнительного» 3.

Новое понимание обществом целей и ценностей образования, смысла образовательных результатов и определяет направления модернизации образования. Более того, в условиях реализаций требований ФГОС нового поколения изменились требования к характеру и содержанию подготовки выпускника школы, поскольку нынешние образовательные результаты не могут в полной мере стать основой для успешного самоопределения и реализации жизненных устремлений выпускников школы. В этой связи перед общеобразовательной школой встал вопрос о необходимости создания новой образовательной среды, новых организационных форм образовательного процесса и методов обучения с использованием информационных и коммуникационных технологий (ИКТ) 4, 7.

Впрочем, эти положения ФГОС можно считать ответом на требования и потребности образования.

Обсуждению проблемы преподавания школьного курса информатики и методической системе обучения этому предмету посвящены многие работы зарубежных $12,14,15$, 18, и отечественных 9, 10, 11 ученых, специалистов и методистов. Существует обширная литература по результатам обсуждения проблемы преподавания информатики в трудах по итогам работы международных конференций 13, 16, 17 (например, SIGCSE, ICER, ISSEP, ACM Transactions on Computing Education и др.).

По мнению М.М. Абдуразакова, С.В. Зенкиной, А.А. Кузнецова, М.П. Лапчика, В.М. Монахова, М.А.Сурхаева, В.В. Фирсова, Е.К. Хеннера и др., традиционная (классно-урочная) система образования не испытывала значительных потребностей в применении новых средств ИКТ в образовательном процессе. Однако, модернизация образования и ориентация учебного процесса на достижение новых образовательных результатов (требования нового ФГОС), не могут быть эффективно и полноценно сформированы в рамках прежней образовательной среды и традиционных методов, организационных форм и средств образовательного процесса.

Ощутимые изменения в результатах образования возможны только при переходе к новой информационно-образовательной среде, и только в этой среде инновационные организационные формы, новые методы и соответствующие им средства обучения, отвечающие потребностям этой среды, позволяют существенно повлиять на достижение современных образовательных результатов 2,8 .

Очевидно, что методические системы обучения, реализуемые и развиваемые в новой ИОС, приобретают новые черты, новый характер взаимодействия своих компонентов. Эти изменения обусловлены в настоящее время двумя основными факторами.

Bo-nервых, ориентацией современной школы на новые цели и ценности образования, адекватные запросам и образовательным потребностям нынешнего российского общества.

Во-вторых, формированием новой информационной образовательной среды школы на основе средств ИКТ.

Чтобы обстоятельно рассмотреть влияние этих двух факторов, необходимо понимание функций, возможностей и взаимосвязи компонентов МСО, в какой образовательной среде осуществляется образовательный процесс, какие внешние и внутренние факторы влияют на ИОС, рассматриваемую нами как дидактическое условие реализации 
требований нового ФГОС и рабочее исследовательское поле профессиональной и педагогической деятельности преподавателя.

Таким образом, одним из путей модернизации образования, придания образовательному процессу инновационного характера является создание новой образовательной среды как часть современного информационно-образовательного и культурнопросветительского пространства.

Методическую систему обучения информатике интересуют следующие вопросы:

- создания ресурсов информатизации образования (ИО), их правового оформления;

- $\quad$ продуктивного использования этих ресурсов, внедрения их в обучение предмета информатики в качестве его средств;

- развития информационной компетентности и личной информационной культуры субъектов обучения через изучение предмета и самостоятельную работу с ресурсами ИО.

Как часть внешней среды для методической системы обучения информатике информатизация образования находится с ней в существенном взаимовлиянии, что получает отражение во все компонентах данной системы - целях, методах, содержании и т.д. Она является не только одним из основных источников образовательных ресурсов, учебнообразовательных технологий, но и источником методики их создания и внедрения.

Как внутренний фактор методической системы обучения информатике - это проекция, образ ИО и методической системы ИО в этой системе:

- отражение в целях и результатах обучения;

- $\quad$ отражение в содержании и методике (подходах, принципах, методах);

- отражение средств ИО в средствах обучения и методике использования (потребления) ресурсов ИО;

- отражение в личностных результатах, в субкультуре работы с информацией и ресурсами ИО.

По мнению Ю.Г. Коротенкова, информационный характер современной образовательной среды - одно из важнейших ее свойств, поскольку оно инвариантно вне зависимости от толкования этого понятия, то «... в век глобальной информатизации признание этого свойства является необходимым для понимания и исследования содержания образовательной среды. Поэтому часто образовательную среду называют информационной образовательной средой (ИОС) и рассматривают ее именно в этом контексте», 5.

ИОС - представление образовательной среды в информационной форме, ее образ в информационных средствах, ресурсах, технологиях, обучающих и управляющих системах, выраженных в документированной форме и методически адаптированных для образования. Основная цель ИОС - обеспечение перехода образования в новое качество: в состояние, соответствующее информационному обществу 1.

Следовательно, образовательная среда определяется как совокупность факторов, определяющих обучение и развитие личности, социокультурные и экономические условия общества, влияющие на образование, характер информационных и межличностных отношений, взаимодействия с социальной средой.

На наш взгляд, ИОС, как продукт информатизации образования, является интегрированным средством реализации образовательного процесса и образовательного взаимодействия, является непосредственным участником межсистемного взаимодействия и диалога субъектов образования.

Качество результатов информатизация образования не определяется количеством внедренных IT-технологий, соответственно, сущность и новизна современной ИОС определяется не только и не столько включением в ее состав новых компонентов, но, прежде всего, эффективностью обеспечения процесса реализации требований нового ФГОС, 
ориентацией ИОС на достижение новых образовательных результатов. Это потребует не только новых компонентов, но и нового взгляда на методическую систему обучения, развитие структуры среды и взаимосвязей ее компонентов. Состав и взаимосвязь компонентов ИОС должны иметь гибкую структуру и функционал, адаптирующиеся к особенностям конкретного содержания обучения, потребностям и способностям обучаемых, то есть иметь открытую архитектуру реализации.

\section{БИБЛИОГРАФИЧЕСКИЕ ССЫЛКИ}

1. Абдуразаков М.М., Цветкова О.Н., Миронова И. В. Представление информационнообразовательной среды в образовательном киберпространстве. [Текст]. Историческая и социально-образовательная мысль. 2016. Том. 8. № 6. Часть 1. -C. 173-179. DOI: http://dx.doi.org/10.17748/2075-9908-2016-8-6/1

2. Абдуразаков, М.М., Ниматулаев М.М., Сурхаев М.А. Развитие методической системы подготовки учителя информатики к использованию средств информационных и коммуникационных технологий. Информатика и образование. №12. 2008.-С. 101-105.

3. «Концепция Федеральной целевой программы развития образования на 2016-2020 годы» Утверждена распоряжением Правительства Российской Федерации от 29 декабря 2014 г. № 2765-p (Режим доступа: http://government.ru/media/files/mlorxfXbbCk.pdf)

4. Коротенков, Ю.Г. Информатизация образования как социальный процесс. // А.Ю. Лазебникова, Ю.Г. Коротенков и др. - М.: ИСМО РАО. - 2010. - 60 с.

5. Коротенков Ю.Г. Учебное пособие «Информационная образовательная среда основной школы». М.: Академия АйТи, 2010. - 152 с.

6. Кузнецов, А.А. Реализация требований нового ФГОС в практике школьного образования // Информатика и образование. - №5. - 2013- С. 3-5.

7. Кузнецов, А.А. Что значат требования к результатам освоения основных общеобразовательных программ? / А.А. Кузнецов // Стандарт общего образования: на пути к общественному договору. - М.: Просвещение, 2006.

8. Кузнецов А.А. Учебник в составе новой информационно-коммуникационной образовательной среды: методическое пособие. / А.А. Кузнецов, С.В. Зенкина. - М.: Бином «Лаборатория знаний». 2010. -63 с.

9. Кузнецов, А.А., Монахов В.М., Абдуразаков М.М. Какой должна быть программа курса «Теория и методика обучения информатике». // Информатика и образование. № 8 (277). 2016. -С. 3 13.

10. Кузнецов, А.А., Монахов В.М., Абдуразаков М.М. Современная и будущая профессиональная деятельность учителя информатики. // Информатика и образование. № 5 (274). 2016. -С. 3 12.

11. Хеннер Е.К. "Школьная информатика. Зарубежный опыт". Сибирские педагогические чтения. Красноярск, декабрь, 2014. Режим доступа:

http://www.kspu.ru/upload/documents/2014/12/18/4ddd43842e7f0679d221e52e6ce6152b/he nnershkolnaya-informatika-zarubezhnyij-opyit.pdf

12. Gal-Ezer J., Stephenson C. A Table of Two Countries: Successes and Chellenges in K-12 Computer Science Education in Israel and United States. ACM TOCE, Special Issue on Computing Education in K-12 Schools, 2014.

13. Informatics education: Europe cannot afford to miss the boat / Report of the joint Informatics Europe \& ACM Europe Working Group on Informatics Education. April 2013. http://europe.acm.org/iereport/ACMandIEreport.pdf

14. Hubwieser P., Armoni M., Giannakos M.N., Mittermeir R. T. Perspectives and Visions of Computer Science Education in K-12 Schools. ACM Transactions on Computing Education. - 2014. - V.14. N.2. - P. 7:1-7:9

15. Helen C. Barrett. Researching electronic portfolios and learner engagement: The Reflect Initiative. Journal of Adolescent \& Adult Literacy, 50 (6). 436-449.

16. Report of the Académie des Sciences. Teaching computer science in France. Tomorrow can't wait. MAY 2013. http://www.academie-sciences.fr/activite/rapport/rads_0513gb.pdf

17. The way forward for computing in UK schools. The Royal Academy of Engineering. 2012. Pp. -122. https://royalsociety.org/ /media/education/computing-in-schools/2012-01-12-computing-inschools.pdf 
18. Wilson C. et.al. Running on Empty: The Failure to Teach K-12 Computer Science in the Digital Age. The Association for Computing Machinery. The Computer Science Teachers Association. http://www.acm.org/runningonempty

\section{REFERENCES.}

1. Abdurazakov, M. M., Tsvetkova, O. N., Mironova, I. V. Representation of information educational environment in educational cyberspace. [Text]. Historical and socio-educational Idea. 2016. Tom. 8. No. 6. Part 1. pp. 173-179. DOI: http://dx.doi.org/10.17748/2075-9908-2016-8-6/1

2. Abdurazakov, M.M, Nimatulaev M.M, Surkhayev M.A Development of a methodical system for the preparation of an informatics teacher for the use of information and communication technologies. Informatics and education. No.12. 2008. Pp. 101-105.

3. The concept of the Federal target program of education development for 2016-2020. Approved by the decree of the Government of the Russian Federation of 29 December 2014 No. 2765-R. Available at: http://government.ru/media/files/mlorxfXbbCk.pdf

4. Korotenkov, Y. G. Informatization of education as a social process. Lazebnikova A. Ju, Korotenkov, Y. G. and others M.: ISMO RAO. -2010. 60 p.

5. Korotenkov Yu.G. Textbook "Information educational environment of the main school." M .: Academy of IT, 2010. 152 p.

6. Kuznetsov, A. A. the Implementation of the requirements of the new GEF in the practice of school education. Informatics and education. No. 5. 2013. Pp. 3-5.

7. Kuznetsov, A. A. What are the requirements to results of mastering basic educational programs? A. A. Kuznetsov. Standard General education: toward a social contract. M.: Education, 2006.

8. Kuznetsov A.A. Textbook as part of a new information and communication educational environment: a methodical guide. A.A. Kuznetsov, S.V. Zenkina. M.: Binom "Laboratory of Knowledge". 2010. $63 \mathrm{p}$

9. Kuznetsov, A. A., Monakhov V. M., Abdurazakov M. M. What should be the program of the course "Theory and methodology of teaching Informatics". Informatics and education. No. 8 (277). 2016. pp. 3-13.

10. Kuznetsov, A. A., Monakhov V. M., Abdurazakov M. M. current and future professional activity of teachers of Informatics. Informatics and education. No. 5 (274). 2016. pp. 3-12.

11. Henner E. K. "School Informatics. Foreign experience". Siberian pedagogical readings. Krasnoyarsk, December, 2014. Available at:

http://www.kspu.ru/upload/documents/2014/12/18/4ddd43842e7f0679d221e52e6ce6152b/he nnershkolnaya-informatika-zarubezhnyij-opyit.pdf

12. Gal-Ezer J., Stephenson C. A Table of Two Countries: Successes and Chellenges in K-12 Computer Science Education in Israel and United States. ACM TOCE, Special Issue on Computing Education in K-12 Schools, 2014.

13. Informatics education: Europe cannot afford to miss the boat. Report of the joint Informatics Europe \& ACM Europe Working Group on Informatics Education. April 2013. Available at: http://europe.acm.org/iereport/ACMandIEreport.pdf

14. Hubwieser P., Armoni M., Giannakos M.N., Mittermeir R. T. Perspectives and Visions of Computer Science Education in K-12 Schools. ACM Transactions on Computing Education. 2014. V.14. N.2. P. 7:1-7:9

15. Helen C. Barrett. Researching electronic portfolios and learner engagement: The Reflect Initiative. Journal of Adolescent \& Adult Literacy, 50 (6). 436-449.

16. Report of the Académie des Sciences. Teaching computer science in France. Tomorrow can't wait. MAY 2013. Available at: http://www.academie-sciences.fr/activite/rapport/rads_0513gb.pdf

17. The way forward for computing in UK schools. The Royal Academy of Engineering. 2012. Pp. 122. Available at: https://royalsociety.org/ /media/education/computing-in-schools/2012-01-12computing-in-schools.pdf

18. Wilson C. et.al. Running on Empty: The Failure to Teach K-12 Computer Science in the Digital Age. The Association for Computing Machinery. The Computer Science Teachers Association. Available at: http://www.acm.org/runningonempty

\section{Информация об авторе:}

Абдуразаков Магомед Мусаевич, доктор педагогических наук, доцент, ведущий

\section{Information about the author:}

Magomed M. Abdurazakov, Doctor of Pedagogical Sciences, Associate Professor, Leading Re- 
научный сотрудник, Центр теории и методики обучения математике и информатике, Институт стратегии развития образования Российской академии образования,

г. Москва, Россия

abdurazakov@inbox.ru

Ниматулаев Магомедхан Магомедович, доктор педагогических наук, профессор, кафедра бизнес-информатики, Финансовый университет при правительстве РФ,

г. Москва, Россия

Аскеров Алаутдин Садитдинович, кандидат педагогических наук, доцент, кафедра информатики, Дагестанский государственный педагогический университет,

г. Махачкала, Россия

Получена: 07.05.2017

Для цитирования: Абдуразаков М.М., Ниматулаев М.М., Аскеров А.С. Образовательная среда как дидактическое условие обеспечения эффективности обучения и развития личности. Историческая и социальнообразовательная мысль. 2017. Том. 9. № 3. Часть 1. с. 115-120.

doi: $\quad 10.17748 / 2075-9908-2017-9-3 / 1-115-$ 120. searcher, Center of Theory and Methodology of Teaching Mathematics and Informatics, Institute for Strategy of Education Development of the Russian Academy of Education,

Moscow, Russia

abdurazakov@inbox.ru

Magomedhan M. Nimatulaev, Doctor of Pedagogical Sciences, Professor, Department of Business Informatics, Financial University under the Government of the Russian Federation, Moscow, Russia

Alautdin S. Askerov, Candidate of Pedagogical Sciences, Associate Professor, Department of Informatics, Dagestan State Pedagogical University,

Makhachkala, Russia

Received: 07.05.2017

For citation: Abdurazakov M.M., Nimatulaev M.M, Askerov A. S.

Educational environment as a didactic condition for ensuring the effectiveness of education and personal development. Historical and Social Educational Idea. 2017. Vol . 9. no.3. Part. 1. Pp. 115-120.

doi: 10.17748/2075-9908-2017-9-3/1-115-120. (in Russian) 